\title{
Comparação de métodos da análise de estabilidade e modelagem estatística para previsão do fator de segurança do talude
}

Os estudos geotécnicos são de extrema importância mediante a qualquer obra a ser realizada, seja em fundações de prédios, em terraplenagens ou em cortes e aterros. Em relação a estabilidade de taludes e seu uso costumeiro em obras da construção civil, não é diferente, sendo necessário analisar e programar ações no solo, uma vez que as consequências causadas pela ruptura em taludes são na maioria das vezes imensuráveis. Este presente trabalho tem como objetivo realizar uma análise sobre a variação dos fatores de segurança obtidos por diferentes métodos ante a simulação de variações de parâmetros de um talude e, a partir desses resultados, obter equações capazes de fornecer o fator de segurança do talude a partir dos valores de tais parâmetros. Para tal, foi utilizado um talude localizado em São Luís/MA. Os parâmetros considerados foram coesão, ângulo de atrito, peso específico do solo e altura do talude. Os cálculos de fatores de segurança pelos métodos de Bishop, Fellenius e Morgenstern-Price foram realizados com a utilização de um software de análise de estabilidade de taludes chamado Geo5. Os resultados das simulações mostraram alta correlação e convergência dos valores obtidos pelos três métodos. Por fim, as equações obtidas apresentaram resultados estatísticos satisfatórios de previsibilidade do fator de segurança, demonstram potencial de serem utilizadas em anteprojetos.

Palavras-chave: Estabilidade de Taludes; Geo5; Fator de Segurança.

\section{Comparison of methods of stability analysis and statistical modeling for slope safety factor forecasting}

\begin{abstract}
Geotechnical studies are of extreme importance through any work to be carried out, whether on building foundations, earthworks or in cuts and embankments. Regarding the stability of slopes and their usual use in civil construction works, it is no different, being necessary to analyze and program actions on the ground, since the consequences caused by the rupture in slopes are most of the times immeasurable. This work aims to carry out an analysis on the variation of the safety factors obtained by different methods before simulating variations in the parameters of a slope and, from these results, to obtain equations capable of providing the safety factor of the slope from of the values of such parameters. For this, a slope located in São Luis / MA was used. The parameters considered were cohesion, friction angle, specific weight of the ground and height of the slope. The calculations of safety factors by the methods of Bishop, Fellenius and Morgenstern-Price were performed with the use of software for the analysis of slope stability called Geo5. The results of the simulations showed a high correlation and convergence of the values obtained by the three methods. Finally, the equations obtained showed satisfactory statistical results of predictability of the safety factor, showing the potential to be used in preliminary projects.
\end{abstract}

Keywords: Slope Stability; Geo5 Software; Safety Factor.

Topic: Engenharia Geotécnica

Reviewed anonymously in the process of blind peer.
Received: 05/06/2021

Approved: 26/06/2021
Alisson Corrêa Nunes (iD)

Universidade Federal do Maranhão, Brasil http://lattes.cnpq.br/0992019237218395 http://orcid.org/0000-0003-1112-6103 ac.nunes@discente.ufma.br

Lucas Cristiano Mendes Santos Universidade Federal do Maranhão, Brasil http://lattes.cnpq.br/7115129899386665 http://orcid.org/0000-0003-3658-1495 lucas.cristiano@discente.ufma.br

Rodrigo da Cruz de Araujo

Universidade Federal do Maranhão, Brasil http://lattes.cnpq.br/2600077439764627 araujo.rodrigo@ufma.br
Referencing this:

NUNES, A. C.; SANTOS, L. C. M.; ARAUJO, R. C.. Comparação de métodos da análise de estabilidade e modelagem estatística para previsão do fator de segurança do talude. Revista Ibero Americana de Ciências Ambientais, v.12, n.6, p.246-259, 2021. DOI: http://doi.org/10.6008/CBPC2179-6858.2021.006.0021 


\section{INTRODUÇÃO}

O estudo de solo é um item fundamental para a boa execução de obras na área da engenharia civil. A partir dele, pode-se caracterizar diversos fatores que majoram o sucesso da obra. Seja para executar fundações de edifícios ou residências, para pavimentação de vias ou até mesmo para execução de taludes ou barragens (DUTRA, 2013).

Em relação à execução dos taludes pode-se abordar sobre o seu uso costumeiro nas obras da construção civil, seja residencial a industrial, a exemplo de taludes originados por cortes nas encostas ou taludes artificiais devido a aterros criados para nivelar o solo (GUIDICINI et al., 1983). De acordo com Matos (2009) devido a estes processos de execuções de encostas, o responsável pela obra deve promover um grau de segurança do talude para que este não gere instabilidades que vão desde os escorregamentos até os sinistros, que resultam em movimentação de grandes volumes de solo e rocha, promovendo danos materiais e pessoais, além de paralisação das atividades operacionais, etc.

Segundo Fiori et al. (2009), a escolha do método de estabilização utilizado envolve diversos fatores, como facilidade de acesso, impacto ambiental, dificuldades de construção, disponibilidade de materiais, custo e prazo. Ademais, a complexidade se deve à singularidade de cada situação, à intervenção de condições relacionadas às propriedades dos materiais constituintes e dos agentes interferentes, sejam essas condições geológicas, antrópicas ou geotécnicas (GUIMARÃES, 2012).

A motivação da realização deste estudo pauta-se na importância de se fazer uma avaliação correta dos fatores de segurança que envolvem um talude que sofreu modificações em sua estrutura natural evitando instabilidades futuras, promovendo maior segurança e confiabilidade tanto em obras como na ocupação do solo.

Com base no exposto, este trabalho tem como objetivo realizar uma análise sobre a variação dos fatores de segurança obtidos por diferentes métodos ante a simulação de variações de parâmetros de um talude e, a partir desses resultados, obter equações capazes de fornecer o fator de segurança do talude a partir dos valores de tais parâmetros.

\section{REVISÃO TEÓRICA}

Os taludes são caracterizados por qualquer superfície inclinada de massa rochosa ou solo e podem ser naturais (também chamadas de encostas) ou artificiais (GUIDICINI et al., 1983). A Associação Brasileira de Geologia de Engenharia (ABGE, 2008) aborda que a estabilidade de taludes é definida como um tratamento aplicado à encosta de um terreno natural ou modificado para melhorar as suas características de resistência, para intervir nos fatores de condicionamento relacionados com as propriedades dos seus materiais constituintes, e para desencadear o processo de provocar a sua instabilidade.

Portanto, quando se assume que a encosta pode apresentar instabilidade, medidas de estabilização devem ser tomadas para evitar possíveis desastres (BASSANELI et al., 2015).

Faz-se necessário conhecer algumas características que promovam a estabilidade do talude como os 
parâmetros da resistência do solo e os métodos de estabilização. Existem dois métodos de análise de estabilidade de taludes: Método determinístico para determinar a segurança de encostas em termos de fatores de segurança e métodos de probabilidade, em que garante a segurança com base na possibilidade ou risco de queda (GEORIO, 2000). Neste trabalho, serão abordados apenas métodos determinísticos tais como: Fellenius, Bishop Simplificado e Morgenstern-Prince.

\section{Parâmetros da Resistência do Solo}

A ruptura dos solos está relacionada ao efeito do cisalhamento, e sua resistência pode ser determinada como a tensão máxima de cisalhamento que o solo pode suportar sem sofrer ruptura, ou a tensão de cisalhamento do solo no plano em que a ruptura estiver ocorrendo. Assim quando uma força ultrapassa este limite, ocorre a ruptura da massa de solo (PINTO, 2002).

Machado et al. (1997) sugerem que a resistência do solo é um resultado de uma ação conjunta de dois fatores denominados de ângulo de atrito e coesão, sendo estas propriedades significativas dos materiais, para a discussão de problemas de estabilidade.

De acordo com a figura 1, a tensão cisalhante $(\tau)$, necessária para causar o deslizamento, aumenta conforme o aumento da tensão normal $(\sigma)$. A inclinação da linha da tensão normal e tensão cisalhante define o ângulo de atrito (ф). Quando a tensão normal for igual a zero e a descontinuidade for selada, ou rugosa, será necessário determinar um valor de tensão cisalhante para gerar movimentação, que determinará a coesão no plano de descontinuidade (PINTO, 2002).

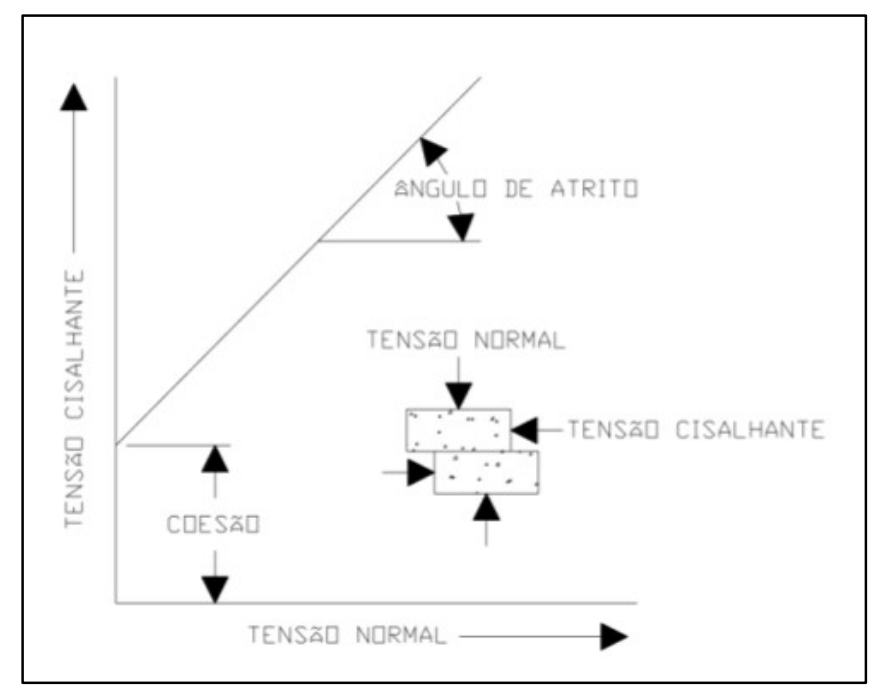

Figura 1: Correlação entre a tensão de cisalhamento necessária para a produção de um escorregamento e a tensão normal atuante. Fonte: Pinto (2002).

\section{Ângulo de Atrito}

O ângulo de atrito pode ser definido, como sendo o ângulo máximo que a força transmitida pelo corpo à sua superfície pode fazer com a normal ao plano de contato sem que ocorra o deslizamento. Enuncia ainda que se atingisse este ângulo, a componente tangencial é maior que a resistência ao deslizamento, que depende da componente normal (PINTO, 2002). 
Pinto (2002), ressalta que existe diferença entre o atrito existente entre dois corpos e o atrito existente nos solos, as partículas de grãos podem se rolar entre si, umas sobre as outras, preenchendo os espaços vazios existentes.

No círculo de Mohr, o ângulo de atrito interno, $\phi$, é caracterizado pelo coeficiente angular da reta que passa pela origem das coordenadas cartesianas, onde $y=\tau$ e $x=\sigma$, sendo denominada de envoltória de Mohr ou linha de ruptura, a reta que representação do limite entre as regiões estáveis e instáveis. Na figura 2, observa-se que o círculo I localiza-se na região estável, enquanto o círculo II tangencia a envoltória, alcançando a condição de ruptura (FIORI et al., 2015).

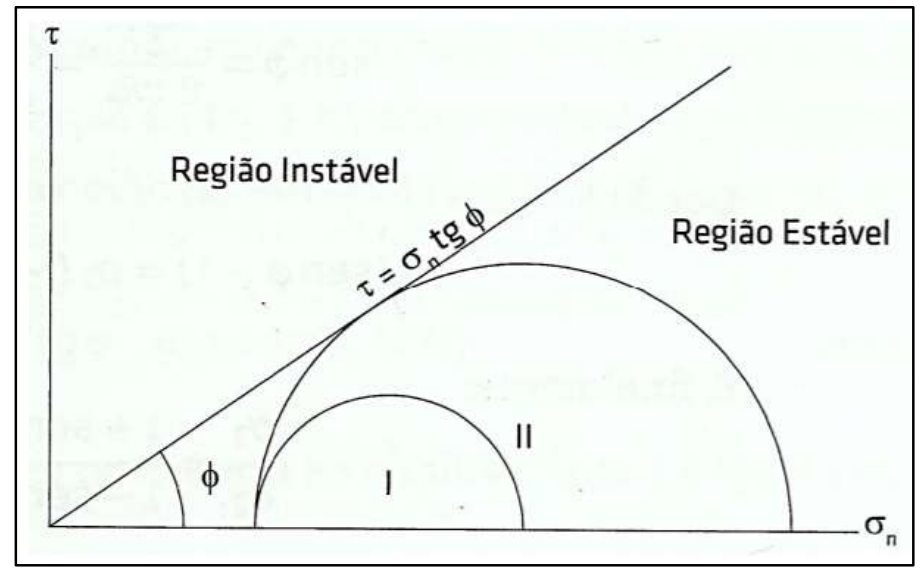

Figura 2: Envoltória de Mohr-Coulomb e ângulo de atrito interno. Fonte: Fiori et al. (2015).

\section{Coesão}

A atração química entre as partículas pode provocar uma resistência independente da tensão normal atuante no plano, constituindo uma coesão real, como se tivesse uma "cola" estivesse sido aplicada entre os grãos do solo (PINTO, 2002).

Machado et al. (1997), exemplificam que a coesão consiste na parcela de resistência de um solo que existe livremente de quaisquer tensões aplicadas e que se mantém, ainda que não necessariamente em logo prazo, caso todas as tensões aplicadas ao solo fossem removidas. Já solos não coesivos, aqueles que não possuem coesão, como areias puras e pedregulhos, esfarelam-se ao serem submetidos a tensões, pois estes apenas possuem uma coesão aparente.

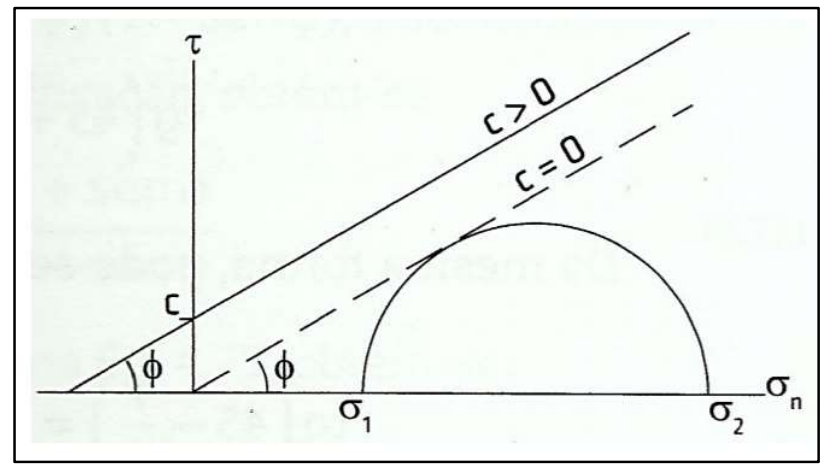

Figura 3: Efeito da coesão no círculo de Mohr-Coulomb. Fonte: Fiori et al. (2015).

De acordo com a figura 3, o efeito positivo da coesão no solo no círculo de Mohr-Coulomb, 
mostrando que quando a coesão é nula $(c=0)$, acontece a ruptura, porém com a presença de coesão $(c>0)$, com as mesmas tensões, o mesmo solo não sofre ruptura, pois o mesmo irá se localizar na região de estabilidade. (FIORE, 2015).

\section{Definição de Fator de Segurança}

Os métodos de cálculo para definir a estabilidade de taludes são feitos com o intuito de garantir que as forças resistentes são suficientemente maiores do que as forças atuantes no sentido de causar a ruptura do maciço (DAS, 2013). A maior parte dos métodos de cálculo utilizam as definições de fator de segurança para obtenção desses dados, sendo que os parâmetros básicos para isso são as características das forças cisalhantes, a poro pressão causada pela água e as propriedades físicas e geométricas do talude em questão (DUNCAN et al., 2014). O FS é definido como o resultado da divisão entre a resistência e a tensão cisalhante, representada na Equação 1 (ABRAMSON et al., 2002).

$$
F S=\frac{\tau_{r}}{\tau_{d}}
$$

Em que, FS é o fator de segurança. $\tau_{r}$ é a tensão de cisalhamento.

$\tau_{d}$ é a resistência ao cisalhamento.

A NBR 11682 (ABNT, 2009) define os fatores de segurança em função do impacto de potenciais rupturas em relação ao envolvimento de vidas humanas e perdas materiais, apresentados na Tabela 1.

Tabela 1: Fator de segurança em função dos riscos à vida humana e perdas materiais.

\begin{tabular}{l|c|c|c}
\hline $\begin{array}{l}\text { Danos à vidas } \\
\text { humanas } \\
\text { e ambientais }\end{array}$ & Alto & Médio & Baixo \\
\hline Alto & 1,5 & 1,5 & 1,4 \\
\hline Médio & 1,5 & 1,4 & 1,3 \\
\hline Baixo & 1,4 & 1,3 & 1,2 \\
\hline
\end{tabular}

Fonte: ABNT (2009).

Segundo a ABNT (2009) o fator de segurança admissível em projeto deve ser maior à medida que se aumenta o grau de incertezas referentes aos parâmetros do solo, custos de recuperação, e perda de vidas humanas. Por definição, valores de FS iguais a 1 correspondem à ocorrência de ruptura, valores maiores que 1 indicam talude estável e valores menor que 1 não possuem significado físico (GERSCOVICH, 2016).

\section{Métodos de Equilíbrio Limite}

Para os métodos de equilíbrio limite, as análises dos resultados obtidos são comumente apresentadas em termos de um fator de segurança que indica a razão entre a resistência disponível e a resistência mobilizada (DUNCAN et al., 2014). Segundo Abramson et al. (2002) ao selecionar o método para analisar a estabilidade do talude é necessário seguir os 4 passos a seguir: 1 - A tentativa de visualizar a provável forma das superfícies de deslizamento deve ser dada à existência de grandes descontinuidades, superfícies de deslizamento existentes, estratificação, não homogeneidade, trincas de tensão e juntas 
abertas; 2 - Distinguir claramente os primeiros deslizamentos e possíveis movimentos renovados ao longo das superfícies deslizantes existentes. Confiar apenas na resistência residual ao longo das partes das superfícies de deslizamento assumidas que correspondem às zonas de cisalhamento existentes ou anteriores; 3 - Tomar decisões sobre os valores relativos aos fatores de segurança no que diz respeito à coesão e friç̧ão. Levar em consideração as condições de infiltração, submersão e rebaixamento, quando apropriado; 4 - Tomar decisões quanto ao uso de tipos de análise de estresse total ou efetivo.

\section{Método de Fellenius}

Segundo Silva (2011), o método de Fellenius, também intitulado de método Sueco, é o mais simples de todos os métodos das fatias pois é o único que estabelece uma equação de primeiro grau para determinar o fator de segurança, não sendo este, portanto, um método iterativo. Gerscovich (2016) afirma que o método Sueco é realizado nas direções normal e tangencial à superfície de ruptura através do equilíbrio de forças em cada fatia. Na figura 4 observa-se as forças atuantes no corte de Fellenius.

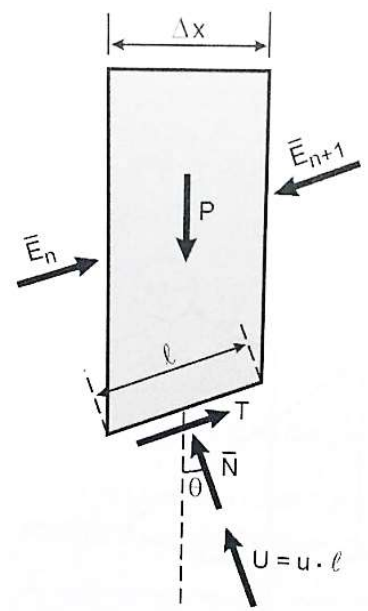

Figura 4: Corte de Fellenius. Fonte: Massad (2010).

Em que,

$$
\bar{N}+U=P \cdot \cos \theta
$$

$\mathrm{Ou}$

$$
\bar{N}=P \cdot \cos \theta-u \cdot \Delta x \cdot \sec \theta
$$

Portanto, o fator de segurança é definido como:

$$
F S=\frac{\sum\left[c^{\prime} \cdot \ell+(P \cdot \cos \theta-u \cdot \Delta x \cdot \sec \theta) \cdot \operatorname{tg} \varphi^{\prime}\right]}{\sum(P \cdot \operatorname{sen} \theta)}
$$

Além disso, Gerscovich (2016) ressalta as seguintes características deste método: É conservativo, ou seja, tende a fornecer baixos valores de FS; Em círculos muito profundos, e quando os valores de poropressão são elevados, o método tende a fornecer valores pouco confiáveis; Nas lamelas localizadas na região estabilizante, onde $\theta$ é negativo, $\bar{N}$ torna-se negativo, devendo então ser tomado como nulo.

Todavia, este método possui algumas falhas. De acordo com Massad (2010) o método Sueco não leva 
em consideração as forças resultantes das pressões neutras. Por serem forças horizontais apresentam componentes na direção normal à base das lamelas.

\section{Método de Bishop Simplificado}

O método de Bishop foi desenvolvido, a priori, para analisar superfícies circulares. No entanto, sua aplicabilidade é validada para superfícies não circulares também. Tal método ignora as forças atuantes no corte entre as fatias como exposto na figura 5. Ressalta-se que os resultados positivos encontrados neste método para o fator de segurança motivaram o estudo mais aprofundado de outros pesquisadores (SILVA, 2011).

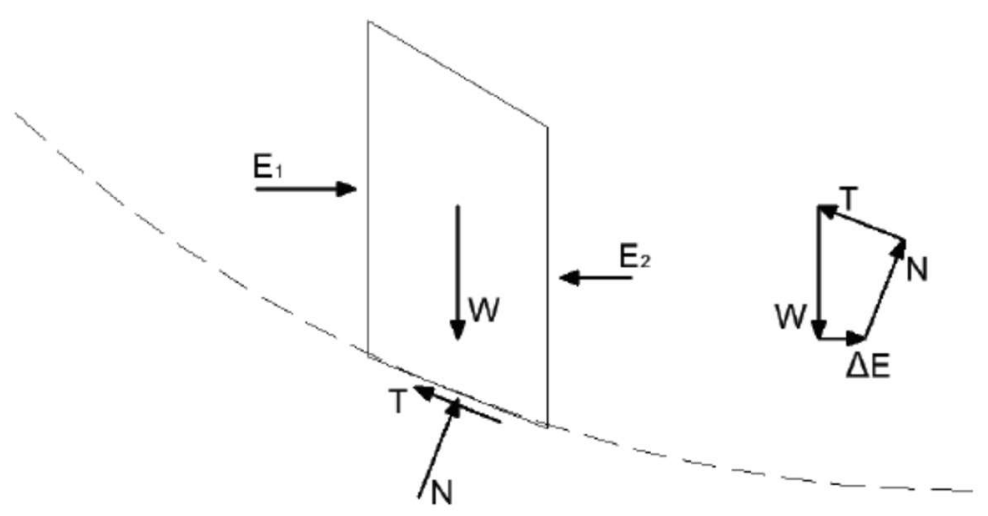

Figura 5: Fatia genérica e polígono de forças. Fonte: Silva (2011).

Massad (2010) diz que no método de Bishop o equilíbrio de forças em cada fatia é feito nas direções verticais e horizontais conforme indica a Figura 6.

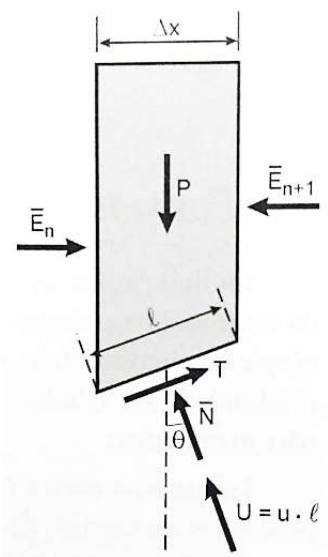

Figura 6: Lamela de Bishop. Fonte: Massad (2010).

Desta forma,

$$
\begin{aligned}
& (\bar{N}+U) \cdot \cos \theta+T \cdot \operatorname{sen} \theta=P \\
& \text { Tendo em vista que } T=\frac{1}{F S}\left(c^{\prime} l+\bar{N} \cdot \operatorname{tg} \varphi^{\prime}\right) \text {, tem-se }
\end{aligned}
$$

$$
\bar{N}=\frac{P-u \cdot \Delta x-\frac{c^{\prime} . \Delta x \cdot \operatorname{tg} \theta}{F S}}{\cos \theta+\frac{\operatorname{tg} \varphi^{\prime} \cdot \operatorname{sen} \theta}{F S}}
$$


Portanto, para o método de Bishop Simplificado FS é definido como:

$$
F S=\frac{\sum\left[c^{\prime} \cdot \ell+\frac{P-u \cdot \Delta x-\frac{c^{\prime} \cdot \Delta x \cdot \operatorname{tg} \theta}{F S}}{\cos \theta+\frac{\operatorname{tg} \varphi^{\prime} \cdot \operatorname{sen} \theta}{F S}} \cdot \operatorname{tg} \varphi^{\prime}\right]}{\sum(P \cdot \operatorname{sen} \theta)}
$$

Gerscovich (2016) fala que como $\bar{N}$ é função de FS, o cálculo do fator de segurança é feito de forma iterativa: arbitra-se um valor inicial $\mathrm{FS}_{1}$, e da expressão extrai-se um novo valor $\mathrm{FS}_{2}$, que é comparado com o inicial. A convergência do processo ocorre quando o valor calculado é igual ao utilizado inicialmente. Em geral, usa-se o FS obtido pelo método Sueco como primeira aproximação.

\section{Método de Morgentern-Price}

O método de Morgenstern-Price é um método rigoroso de análise de estabilidade de taludes, que admite superfície de ruptura qualquer e satisfaz todas as condições de equilíbrio estático (TONUS, 2009). 0 que corrobora com a ideia de Silva (2011) que o método realiza todas as condições de equilíbrio, fazendo este pertencer ao grupo dos métodos rigorosos. Portanto, a aplicação do método acontece nas equações diferenciais que governam o equilíbrio de momentos e o equilíbrio de forças numa fatia (FERREIRA, 2012). Gercovich (2016) faz uma consideração acerca da relação entre a inclinação da resultante ( $\theta$ ) e a superfície de ruptura através da seguinte equação:

$$
T=\lambda \mathrm{f}(\mathrm{x}) E(7)
$$

Em que T e $E$ são as forças verticais e horizontais entre as fatias, $\lambda$ é um parâmetro escalar determinado a partir da solução do cálculo do fator de segurança e $f(x)$ uma função arbitrária. Duncan et al. (2014) explicam que a função f(x) exige uma análise prévia de como a inclinação das forças entre as fatias do talude sofre variação haja vista que quando se utiliza $f(x)=0$, o FS é idêntico ao método de Bishop.

\section{METODOLOGIA}

A metodologia do trabalho constou primeiramente da realização de uma revisão bibliográfica referente ao tema. Esta etapa envolveu o levantamento de fontes, cruzamento de referências obtidas da literatura e estudos de numerosos artigos técnico-científicos em periódicos e um número restrito de livros foi igualmente consultado, com a finalidade de proporcionar o embasamento teórico necessário para se atingir os objetivos da pesquisa.

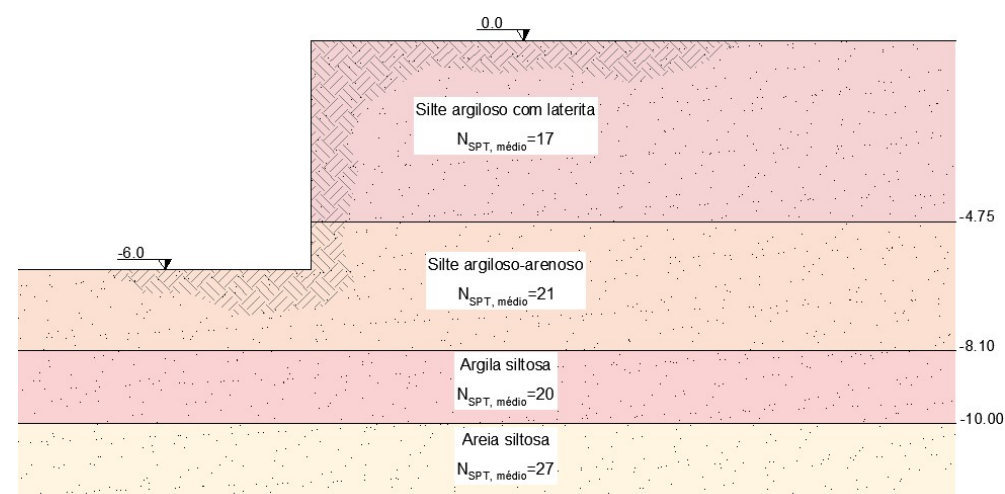

Figura 7: Perfil Estratigráfico do Talude estudado. Fonte: Oliveira et al. (2021). 
A análise utilizará como referência um talude real no município de São Luís/MA, o qual já foi anteriormente utilizado em um estudo de Oliveira et al. (2021). A análise será feita por meio do Software GEO5, utilizando a teoria do equilíbrio limite (TEL).

\section{Planejamento da Pesquisa}

O estudo da estabilidade dos taludes será realizado por meio de métodos baseados na Teoria do Equilíbrio Limite, mais comumente utilizada nos problemas de estabilidade e caracterizada pela formação de uma superfície de ruptura plana, circular ou mista, onde a razão entre a tensão cisalhante e a tensão normal atinge um valor crítico.

Esta pesquisa foi planejada em quatro etapas principais: Revisão Bibliográfica, Pesquisa por Software e Análise Paramétrica. Para realizar a análise paramétrica e, posteriormente, obter equações capazes de prever o fator de segurança a partir de parâmetros relativos às características do talude foram realizadas diversas simulações, variando individualmente cada um deles. Assim, um fator que foi estudado levando em consideração à geometria, que é a altura (h). Em relação aos parâmetros do solo, foram utilizados valores referentes a ângulo de atrito $(\phi)$, coesão $(c)$, e peso específico natural $(\gamma n)$ do solo. Os dados usados no estudado, previamente estimados, podem ser verificados na tabela 2. Para adotar tais valores, tomou-se como referência os valores apontados por Oliveira et al. (2021) para o mesmo perfil e, a partir deles, promoveu-se variações para mais e para menos a cada parâmetro.

Tabela 2: Dados usados nas simulações

\begin{tabular}{|l|l|l|l|}
\hline Variáveis Estudadas & Faixa de Valores Definidas \\
\hline Altura $(\mathrm{h})$ & 4 & 6 & 8 \\
\hline Coesão $(\mathrm{c})$ & 11 & 13 & 15 \\
\hline Ângulo de Atrito $(\phi)$ & 17 & 19 & 21 \\
\hline Peso Específico Natural $(\lambda \mathrm{n})$ & 17 & 19 & 21 \\
\hline
\end{tabular}

O cruzamento dessas variáveis resultou em 81 combinações diferentes. Estas foram inseridas em um programa computacional (GEO5) que determinou o fator de segurança otimizado para cada situação, considerando a superfície de ruptura crítica. Nesta pesquisa foram adotados três métodos de cálculo do fator de segurança (FS) (perfazendo então 243 simulações, ou seja, 243 fatores de segurança): Método Ordinário (Fellenius), Simplificado de Bishop, e Morgenstern-Price, diferenciando-se entre si pelas hipóteses simplificadoras adotadas em cada um deles.

A partir dos resultados das simulações foram propostas equações que pretendem prever os fatores de segurança por cada método a partir apenas do conhecimento dos valores das variáveis (parâmetros do talude) supracitadas. Para obter tais equações utilizou-se regressão linear múltipla, correlacionando a variável de resposta (variável dependente, neste caso o fator de segurança) com variáveis independentes (parâmetros do talude). Para verificar quão bem os modelos representam os dados experimentais, recorrese a verificação do coeficiente ajustado de determinação (R2 ajustado) e ao erro padrão de estimativa ( $\varepsilon p$ ). 


\section{Software Geo5}

O software Geo5 é um programa da empresa FINE Engineering Software, de origem na República Tcheca, em que apresenta um pacote de programas que fornece a solução para a maioria dos problemas na área de geotecnia. Possui interface intuitiva e simples o que ajuda na execução das atividades, já que este programa não exige um treinamento especial.

Em relação aos programas que o Geo5 compreende, utilizou-se neste trabalho o módulo "Estabilidade de Taludes". Neste módulo usa-se é possível realizar verificações de estabilidade de taludes por diversos métodos, desde os métodos conservadores como o de Fellenius aos métodos rígidos como o de Morgenstern-Price. Nas simulações, o software oferece a opção de otimização, na qual são realizadas iterações que possibilitam a determinação da superfície crítica e, consequentemente, do fator de segurança mínimo para o talude.

\section{RESULTADOS E DISCUSSÃO}

Nesta análise foram realizadas comparações entre os 3 métodos de cálculo utilizados neste estudo. Ademais, foi realizado uma analogia entre os resultados obtidos nas simulações.

\section{Comparação entre os métodos utilizados}

Inicialmente, são apresentadas a seguir as comparações (dois a dois) entre os resultados obtidos para os diferentes métodos de análise de estabilidade adotados.

\section{Fellenius x Bishop Simplificado (Fatores de Segurança)}

Esta análise realizou-se ordenando os valores de FS em ordem numérica, resguardando os valores das variáveis e por consequência comparando os valores dos fatores de segurança obtidos através do método de Fellenius com o método de Bishop Simplificado.

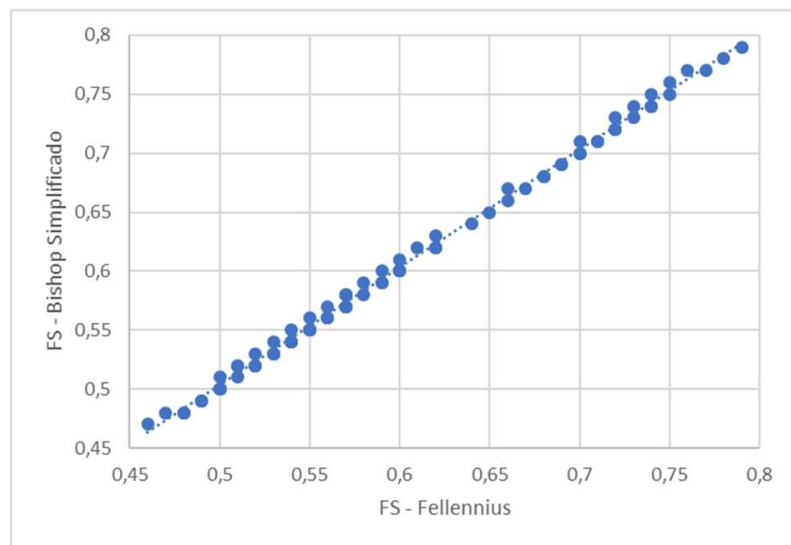

Gráfico 1: Comparação entre os métodos de Bishop Simplificado e o Método de Fellenius.

Depreende-se do gráfico 1 que há um grande equilíbrio e convergência entre os FS. Entretanto, em alguns casos o método do cálculo de Bishop Simplificado apresenta valores de FS ligeiramente superiores em relação ao método de Fellenius. 


\section{Fellenius x Morgenstern-Price (Fatores de Segurança)}

Usando o mesmo raciocínio do item anterior ordenou-se os valores de FS em ordem numérica, resguardando os valores das variáveis e por consequência comparando os valores dos fatores de segurança obtidos por meio do método de Fellenius com o método de Morgenstern-Price.

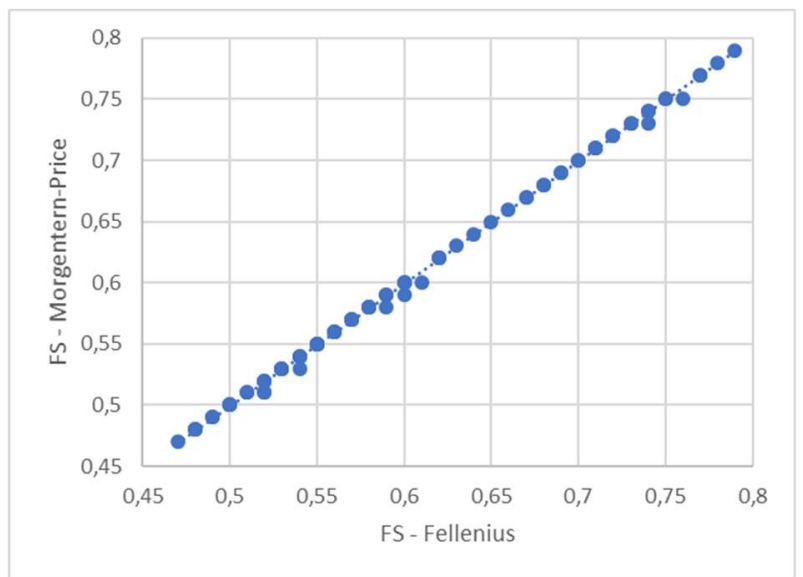

Gráfico 2: Analogia entre os métodos de Fellenius e de Morgenstern-Price.

Nesta comparação observa-se no gráfico 2 que a convergência permanece muito elevada, porém se nota um desequilíbrio ligeiramente maior entre os dois métodos. Apesar da diferença ser pequena, a tendência é a favor de valores maiores do fator de segurança para o método de Morgenstern-Price em relação aos obtidos pelo método de Fellenius.

\section{Bishop Simplificado x Morgenstern-Price (Fatores de Segurança)}

Por último, continuando as análises ordenou-se os valores de FS em ordem numérica, resguardando os valores das variáveis e por consequência comparando os valores dos fatores de segurança obtidos através do método de Bishop Simplificado com o método de Morgenstern-Price.

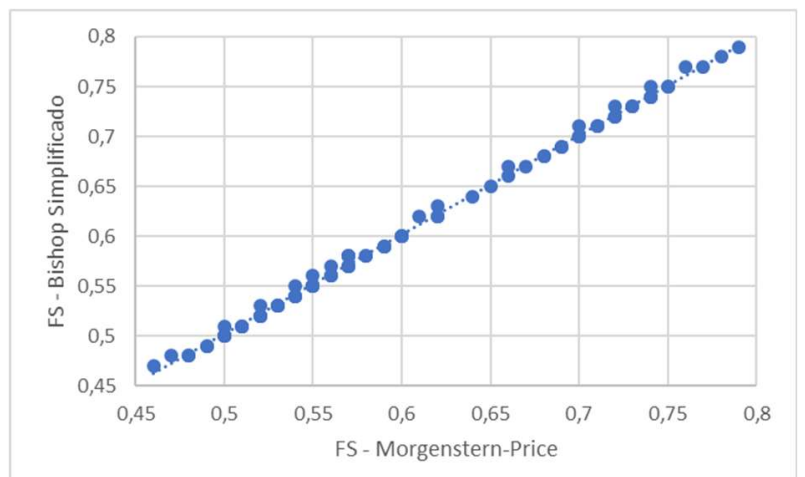

Gráfico 3: Analogia entre os métodos de Bishop Simplificado e de Morgenstern-Price.

Observa-se neste caso que a elevada convergência permanece, havendo uma tendência para valores maiores de FS no método de cálculo de Morgenstern-Price em relação ao método de Bishop Simplificado, ressaltando-se aqui que tais diferenças entre os valores se mantém em uma ordem de grandeza bastante reduzida. 


\section{Modelagem Estatística}

A partir da análise dos dados obtidos nas simulações no programa Geo5, foi realizada uma análise estatística utilizando o programa Excel, para determinar um modelo de regressão linear que indique a influência das variáveis.

Para os modelos de equações gerados, resulta o valor de FS para os métodos de Bishop, Fellenius e Morgenstern-Price, a partir das variáveis utilizadas na pesquisa, sendo elas, peso específico, ângulo de atrito, coesão e altura.

$$
\begin{aligned}
& \mathrm{FS}_{\text {Bishop }}=0,63775-0,01065 \mathrm{Y}+0,00759 \mathrm{Phi}+0,02111 \mathrm{C}-0,04176 \mathrm{H} \\
& \mathrm{FS}_{\text {Fell }}=0,63759-0,01037 \mathrm{Y}+0,00713 \mathrm{Phi}+0,02176 \mathrm{C}-0,04204 \mathrm{H} \\
& \mathrm{FS}_{\text {Morg }}=0,6367-0,01037 \mathrm{Y}+0,00731 \mathrm{Phi}+0,02148 \mathrm{C}-0,04204 \mathrm{H}
\end{aligned}
$$

Os modelos apresentados acima são aplicáveis apenas para taludes a 90, e que tenham toda altura da face constituída por apenas um tipo de solo, por ser baseado no objeto de estudo, não levando em consideração outras possibilidades de inclinações do talude ou de perfis multicamadas. Portanto, são aplicáveis apenas para taludes que contém a mesma geometria.

\section{Modelagem para o modelo de Bishop}

A equação 9apresenta a análise de regressão linear múltipla relacionado à variável dependente FS de Bishop com as variáveis independentes, considerando todos os resultados obtidos durante as simulações efetuadas no programa Geo5.

O coeficiente de determinação $R$ múltiplo é 0,8852 , o $R^{2}$ é 0,7836 , o $R^{2}$ ajustado é 0,7722 e erro padrão é 0,0430 para o FS, conforme indicado na tabela 3. Assim, quanto maior o $\mathrm{R}^{2}$, mais explicativo é o modelo linear, ou seja, melhor ele se ajusta à amostra.

Tabela 3: Estatística de Regressão do FS de Bishop.

\begin{tabular}{ll}
\hline Estatística de regressão & \\
\hline R múltiplo & 0,8852 \\
R-Quadrado & 0,7836 \\
R-quadrado ajustado & 0,7722 \\
Erro padrão & 0,0430 \\
Observações & 81 \\
\hline
\end{tabular}

Observa-se que nesta análise o erro padrão é 0,043, considerado baixo de acordo com as literaturas, devido aos resultados se aproximarem entre si. Considera-se também que o valor de $\mathrm{R}^{2}$ é 0,7836 expresso em termos percentuais de 78,36\% mostrando o quão próximos estão os valores a linha de regressão.

\section{Modelagem para o modelo de Fellenius}

Este modelo, equação 10, representa a análise de regressão linear múltipla relacionado a variável dependente FS de Fellenius, com as variáveis independentes, considerando todos os resultados obtidos durante as simulações efetuadas no programa Geo5.

O coeficiente de determinação $R$ múltiplo é 0,8935 , o $R^{2}$ é 0,7984 , o $R^{2}$ ajustado é 0,7878 e erro 
padrão é 0,0415 para o FS, conforme indicado na tabela 4.

Tabela 4: Estatística de Regressão do FS de Fellenius.

\begin{tabular}{ll}
\hline Estatística de regressão & \\
\hline R múltiplo & 0,8935 \\
R-Quadrado & 0,7984 \\
R-quadrado ajustado & 0,7878 \\
Erro padrão & 0,0415 \\
Observações & 81 \\
\hline
\end{tabular}

Observa-se que nesta analise o erro padrão é 0,0415 , considerado baixo como no caso anterior, devido aos resultados se aproximarem entre si. Considera-se também que o valor de $R^{2}$ é 0,7984 expresso em termos percentuais de $79,84 \%$ aproximando ainda mais os valores a linha de regressão.

\section{Modelagem para o modelo de Morgenstern-Price}

Por fim, a análise de regressão linear múltipla relacionado a variável dependente FS de MorgensternPrice com as variáveis independentes, considerando todos os resultados obtidos durante as simulações efetuadas no programa Geo5 é apresentada no modelo da equação 11.

O coeficiente de determinação $R$ múltiplo é 0,8917 , o $R^{2}$ é 0,7951 , o $R^{2}$ ajustado é 0,7843 e erro padrão é 0,0418 para o FS, conforme indicado na tabela 5 .

Tabela 5: Estatística de Regressão do FS de Morgenstern-Price.

\begin{tabular}{ll}
\hline Estatística de regressão & \\
\hline R múltiplo & 0,8917 \\
R-Quadrado & 0,7951 \\
R-quadrado ajustado & 0,7843 \\
Erro padrão & 0,0418 \\
Observações & 81 \\
\hline
\end{tabular}

Observa-se que nesta análise o erro padrão é 0,0418 , considerado baixo como nos demais casos, evidenciando ainda mais a proximidade dos resultados obtidos através das simulações. Considera-se também que o valor de $\mathrm{R}^{2}$ é 0,7951 expresso em termos percentuais de $79,51 \%$ mostrando o quão próximos estão os valores a linha de regressão.

\section{CONCLUSÕES}

Este estudo baseou-se nas literaturas, em softwares disponíveis no mercado, a identificação dos métodos de estudo e as variáveis que influenciam no fator de segurança no cálculo da estabilidade do talude estudado. A revisão da literatura apontou os principais métodos de cálculo conhecidos, desde o menos rigoroso que é o método de Fellenius aos métodos rigorosos como o método de Morgenstern-Price.

A análise paramétrica permitiu efetuar comparações entre os métodos de cálculo estudados. Observou-se que todos os métodos estudados convergem entre si, com valores de segurança variando a partir da segunda casa decimal. Vale ressaltar que toda a análise usando o programa Geo5 usou a forma otimizada para encontrar os fatores de segurança.

Em relação a modelagem estatística, todos os métodos apresentam valores muito aproximados. Esta 
modelagem permitiu identificar que as análises de erro padrão apresentaram valores baixos o que demonstra consonância com os valores obtidos nas simulações. Ademais, em todas as equações propostas para determinação os fatores de segurança os valores de $R^{2}$ variaram de 0,7836 a 0,7984 evidenciam que estão próximos dos valores da linha de regressão, o que indica bom potencial de preverem adequadamente os fatores de segurança e, dessa maneira, boa adequabilidade de serem aplicadas especialmente em estudos de anteprojetos.

Como sugestões de pesquisas futuras, seria interessante inserir nas simulações e na modelagem estatística variações na inclinação, bem como a possibilidade de haver camadas de solos diferentes sendo cortadas pela face do talude.

\section{REFERÊNCIAS}

ABRAMSON, L. W.; LEE, T. S.; SHARMA, S.; BOYCE, G. M.. Slope stability and stabilization methods. 2 ed. New York: John Wiley \& Sons, 2002.

ABGE. Associação Brasileira de Geologia e Engenharia. Glossário: Geotecnologia Ambiental. 2008.

ABNT. Associação Brasileira de Normas Técnicas. NBR 11682: Estabilidade de Encostas. 2 ed. Rio de Janeiro: ABNT, 2009.

BASSANELI, H. R.; CORRÊA, M. A. M.; SANTO, A. P. C. E.; CRUZ, A. G. E. R. Z. C.; SILVA, B. G. R.; SANTOS, G. L.; MARIOTTO, T. B.. Estabilidade de Taludes. Educação e Sociedade, v.1, n.2, p.1-44, 2016.

DAS, B. M.. Fundamentos de engenharia geotécnica. 7 ed. São Paulo: Cengage Learning, 2013.

DUNCAN, J. M.; WRIGHT, S. G.; BRANDON, T. L.. Soil strength and Slope Stability. 2 ed. New Jersey: Wiley, 2014.

DUTRA, V. A. S.. Projeto de estabilização de taludes e estruturas de contenção englobando dimensionamento geotécnico e estrutural. Monografia (Bacharelado em Engenharia Civil) - Faculdade de Engenharia da Universidade do Estado do Rio de Janeiro, Rio de Janeiro, 2013.

FERREIRA, J. L. F.. Análise de estabilidade de Taludes pelos métodos de Janbu e Spencer. Dissertação (Mestrado em Engenharia Civil) - Universidade do Porto, Porto, 2012.

FIORI, A. P.; CARMIGNANI, L.. Fundamentos de mecânica dos solos e das rochas: aplicações na estabilidade de taludes. 3 ed. São Paulo: Oficina de Textos, 2015.

GEORIO. Fundação Instituto de Geotécnica do Município do Rio de Janeiro. Manual Técnico de Encostas: Análise e Investigação. 2 ed. Rio de Janeiro: GEORIO, 2000.

GERSCOVICH, D. M. S.. Estabilidade de Taludes. 2 ed. São
Paulo: Oficina de Textos, 2016.

GUIDICINI, G.; NIEBLE, C. M.. Estabilidade de taludes naturais e de escavação. 2 ed. São Paulo: Edgard Blucher, 1983.

GUIMARÃES, S. T. L.. Gestão de Áreas de Risco e Desastres Ambientais. Rio Claro, 2012.

MATOS, K. C. A.. Processo de Instabilidade em Taludes Rodoviários em solos residuais arenosos: estudo na rodovia castello branco (sp 280), km 305 à 313. Dissertação (Mestrado em Engenharia Civil) - Universidade de São Paulo, São Carlos, 2009.

OLIVEIRA, A. M.; ARAUJO, R. C.; LUCZYNSKI, M.. Dimensionamento geotécnico de uma estrutura de contenção utilizando software. Revista Ibero Americana de Ciências Ambientais, v.12, n.3, p.224-237, 2021. DOI: http://doi.org/10.6008/CBPC2179-6858.2021.003.0020

MACHADO, S. L.; MACHADO, M. F.. Mecânica do Solos 2: conceitos introdutórios. Universidade Federal da Bahia, Salvador, 1997

MASSAD, F.. Obras de Terra: curso básico de Geotecnia. 2 ed. São Paulo: Oficina de Textos, 2010

PINTO, C. S.. Curso básico de mecânica dos solos em 16 aulas. 2 ed. São Paulo. Oficina de Textos, 2002.

SILVA, J. P. M.. Os métodos de equilíbrio limite e dos elementos finitos na análise de estabilidade de taludes. Dissertação (Mestrado em Engenharia Civil) - Universidade do Porto, Porto, 2011.

TONUS, B. P. A.. Estabilidade de Taludes: avaliação dos métodos de equilíbrio limite aplicados a uma encosta coluvionar e residual da serra do mar paranaense. Dissertação (Mestrado em Construção Civil) - Universidade Federal do Paraná, Curitiba, 2009.

A CBPC - Companhia Brasileira de Produção Científica (CNPJ: 11.221.422/0001-03) detém os direitos materiais desta publicação. Os direitos referem-se à publicação do trabalho em qualquer parte do mundo, incluindo os direitos às renovações, expansões e disseminações da contribuição, bem como outros direitos subsidiários. Todos os trabalhos publicados eletronicamente poderão posteriormente ser publicados em coletâneas impressas sob coordenação da Sustenere Publishing, da Companhia Brasileira de Produção Científica e seus parceiros autorizados. Os (as) autores (as) preservam os direitos autorais, mas não têm permissão para a publicação da contribuição em outro meio, impresso ou digital, em português ou em tradução. 